\title{
Associations between objective and self-reported physical activity and vitamin D serum levels in the US population
}

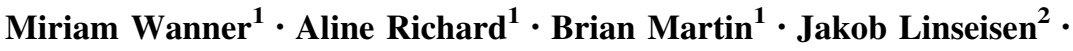 \\ Sabine Rohrmann ${ }^{1}$
}

Received: 6 January 2015/ Accepted: 12 March 2015/Published online: 20 March 2015

(C) Springer International Publishing Switzerland 2015

\begin{abstract}
Purpose Both low levels of vitamin D and of physical activity are associated with all-cause, cancer, and cardiovascular disease mortality. There is some evidence based on self-reported activity levels that physically more active individuals have higher vitamin D serum levels. The aim was to investigate associations between objectively measured and self-reported physical activity, respectively, and vitamin D serum concentrations in the US population.

Methods Data from NHANES 2003-2006 $(n=6,370$, aged $\geq 18$ years) were analyzed using multiple regression analyses. A total of 6,370 individuals aged 18 years and older with valid data on vitamin D serum levels and physical activity were included. Objective physical activity was assessed using accelerometers; self-reported physical activity was based on the NHANES physical activity questionnaire.

Results An increase of 10 min of objectively measured and self-reported moderate-to-vigorous activities per day was associated with an increase in circulating vitamin D of
\end{abstract}

Electronic supplementary material The online version of this article (doi:10.1007/s10552-015-0563-y) contains supplementary material, which is available to authorized users.

Sabine Rohrmann

sabine.rohrmann@ifspm.uzh.ch

1 Division of Chronic Disease Epidemiology, Department of Epidemiology, Epidemiology, Biostatistics and Prevention Institute, University of Zurich, Seilergraben 49, 8001 Zurich, Switzerland

2 Institute of Epidemiology, German Research Centre for Environmental Health (HMGU), Ingolstädter Landstr. 1, 85764 Neuherberg, Germany
$0.32 \mathrm{ng} / \mathrm{ml}(95 \%$ CI $0.17,0.48)$ and of $0.18 \mathrm{ng} / \mathrm{ml}(95 \%$ CI $0.12,0.23)$, respectively. The odds ratio for being vitamin D deficient $(<20 \mathrm{ng} / \mathrm{ml})$ if being insufficiently active compared with being sufficiently active was 1.32 (1.11, 1.57). Associations were not stronger for self-reported outdoor activities compared with indoor activities.

Conclusions Physical activity may be a way to achieve higher vitamin D serum levels in the population. Factors other than sun exposure that may be responsible for higher vitamin $\mathrm{D}$ levels in more active individuals need further investigation.

Keywords 25-Hydroxyvitamin D - Accelerometers · NHANES $\cdot$ Physical activity $\cdot$ USA

\section{Introduction}

Several studies have shown associations between low vitamin D levels and adverse health outcomes: Associations with all-cause [1-6], cancer [5-7], and cardiovascular disease [3-6] mortality were reported. Furthermore, associations between low vitamin D levels and morbidity were described for some types of cancer [8,9], cardiovascular disease [10], and type 2 diabetes [11, 12]. Some of the studies regarding vitamin D and mortality $[3-5,13]$ as well as other outcomes $[10,12,14]$ were based on data of the National Health and Nutrition Examination Survey (NHANES).

At the same time, it is well recognized that physical activity has positive effects on health $[15,16]$. Lower mortality rates in more active individuals have also been reported in the NHANES population [17, 18]. These studies were based on self-reported physical activity. Objective physical activity data from NHANES have mostly 
been analyzed regarding prevalence and patterns of physical activity [19-22].

There are some studies that reported on physical activity as correlates or predictors of vitamin D status [23-28]. Scragg and Camargo [23] reported associations between the frequency of leisure-time physical activity and vitamin D levels in NHANES III based on self-reported physical activity [23]. Specifically, they reported higher vitamin D levels for the same amount of outdoor compared with indoor activities, suggesting that sun exposure may trigger higher vitamin D levels in more active individuals. However, vitamin D levels also increased for higher amounts of indoor activity. In postmenopausal women, recreational physical activity was a predictor for higher vitamin D levels [27]. However, associations were attenuated (but still significant) when adjusting for covariates such as sun exposure, season, vitamin D intake, and waist circumference [24]. Physical inactivity, among other factors such as obesity and low vitamin D dietary and supplement intake, was reported as a major modifiable predictor of low vitamin D status in the USA [25]. Another study including participants from around the world reported vigorous physical activity to be positively correlated with vitamin D levels [26]. In African-American men, who are known to suffer more often from low vitamin D levels than white men, vitamin D concentrations increased with more recreational physical activity [28].

To our knowledge, the present study is the first to use objective physical activity data in combination with vitamin D levels. Furthermore, while Scragg and Camargo [23] used self-reported physical activity data from NHANES III, we analyzed more recent and more detailed self-reported physical activity data from NHANES 2003-06 that also includes information on the average duration in addition to the frequency and intensity.

The aim of this study was to investigate associations between objectively measured as well as self-reported physical activity and vitamin D levels in a large sample of US adults adjusting for a number of potential confounders. Furthermore, stratified analyses according to sex, age, ethnicity, body mass index (BMI), vitamin D supplement intake, and season/region will shed light on potentially different associations in these subgroups.

\section{Materials and methods}

\section{Study population}

The study is based on data from the National Health and Nutrition Examination Survey (NHANES) 2003-2006, two cross-sectional survey cycles (2003/2004 and 2005/2006, respectively) representative of the US civilian non- institutionalized population carried out by the National Center for Health Statistics of the Centers for Disease Control and Prevention. A multistage probability sampling is used to recruit participants. The National Center for Health Statistics Institutional Review Board has reviewed and approved the survey protocol, and written informed consent was obtained from all participants. A detailed description of the surveys has been published elsewhere [29].

For the present analyses, data on serum vitamin D levels were available from blood samples; objective physical activity data were available from accelerometer measures included in the physical examinations. Data of more than 20,000 individuals are available for NHANES 2003-2006. We included 11,183 individuals aged 18 years and older. Of these, 546 individuals were excluded because they only participated in the interview part but not in the mobile examination center part of NHANES. A further 606 individuals were excluded due to missing data on vitamin $\mathrm{D}$ serum levels ( $n=10,031$ ). Finally, 3,661 individuals were excluded because they had not sufficient valid objective physical activity data (see below), leaving 6,370 individuals for analyses.

\section{Measurements}

\section{Physical activity (exposure)}

Objective physical activity was measured in 2003/04 and 2005/06 using the ActiGraph 7164 accelerometer (ActiGraph, Shalimar, FL). This accelerometer has been validated in earlier studies [30-32]. At the medical examination center (MEC), participants aged 6 years and older were asked to wear an accelerometer on their right hip for 7 consecutive days following the examination. An epoch time of $1 \mathrm{~min}$ was used. Individuals were included in the analyses if they had at least 4 days of physical activity data with at least $10 \mathrm{~h}$ per day of accelerometer wear time [20]. For sensitivity analyses, we also analyzed data of individuals with at least 1 day of physical activity data with at least 8 valid hours per day $(n=8,178)$.

The units of measurement of the ActiGraph accelerometer are "counts", filtered accelerometer values summed over a specified epoch time such as $1 \mathrm{~min}$. The value of the counts varies based on the frequency and intensity of the raw acceleration. In order to quantify the duration of physical activity in different intensity levels, the following cut points were used: sedentary (<100 counts/min) [21], light (100-759 counts/min), lifestyle (760-2,019 counts/min) [33], moderate (2,020-5,998 counts/min), and vigorous ( $\geq 5,999$ counts/ min) [19]. Counts/minute, minutes spent in moderate-tovigorous physical activity per day, and physical activity category (achieving $\geq 150$ min per week of moderate-tovigorous activities versus less corresponding to sufficiently 
active versus insufficiently active) were used as exposure variables in the regression models. There is no information regarding where the physical activity took place (indoor/ outdoor).

Self-reported physical activity was based on the NHANES physical activity questionnaire and included the individual activity questions. To our knowledge, this questionnaire is not validated. Only leisure-time physical activity (but not transport-related activity and household activity) was used as these activities correlated best with objectively measured physical activity (data not shown). Participants stating that they engaged in moderate or vigorous leisure-time physical activities reported individual activities including the intensity level, the frequency during the past 30 days, and the average duration. Examples of moderate activities are brisk walking and gardening, examples of vigorous activities running and fast bicycling. Minutes per day were calculated for total leisure-time activities and for moderate and vigorous activities separately. Furthermore, individual activities were categorized as "indoor" and "outdoor" based on discussion with experts that are familiar with the physical activity habits in the USA. Minutes per day spent in indoor and outdoor leisuretime activities were calculated.

\section{Vitamin D (outcome)}

Serum 25-hydroxyvitamin D concentrations were measured at the National Center for Environmental Health using a radioimmunoassay kit (Diasorin Corporation, Stillwater, MN) [34, 35]. A level of $<20 \mathrm{ng} / \mathrm{ml}$ was used to define vitamin D deficiency [36].

\section{Other variables}

Socio-demographic variables [education [36], race/ethnicity [36, 37], gender [37], age [37], marital status, and poverty/ income ratio (PIR)] were considered as potential confounders. Education was dichotomized into post-secondary education (college or higher) versus lower. Ethnicity was categorized as non-Hispanic white, non-Hispanic black, Mexican American, and other. Marital status included living together versus single (including widowed, separated, divorced, and never married). As a measure of socioeconomic status, PIR was categorized as at or above poverty versus below poverty. Season was taken into account based on the time of assessment as higher-latitude regions in the northern states tended to be sampled during the summer months, whereas lower-latitude regions in the south tended to be sampled in the winter months (northern states/summer: May-October; southern states/winter: November-April [3, 4]). There is no more precise variable available for season in NHANES.
Self-reported smoking (current smoker versus current non-smoker) and alcohol intake (no drinks, $\leq 1$ drink per week, and $>1$ drink per week) were used to reflect other health behaviors. Furthermore, milk consumption (daily versus less than daily) with respect to fortification of milk with vitamin D in the USA [36, 38] and the intake of vitamin D supplements (mcg/day) based on detailed information on supplement intake and ingredient information were included.

Regarding health-related variables, BMI [36, 37, 39], general health status [36], hypertension [36, 40], cholesterol [36], and diabetes [11, 12] were taken into account. BMI was based on measured height and weight and categorized as normal weight (BMI $<25 \mathrm{~kg} / \mathrm{m}^{2}$ ), overweight $\quad\left(25 \mathrm{~kg} / \mathrm{m}^{2} \leq \mathrm{BMI}<30 \mathrm{~kg} / \mathrm{m}^{2}\right), \quad$ and obese $\left(B M I \geq 30 \mathrm{~kg} / \mathrm{m}^{2}\right)$. General health status was derived from questionnaire data and coded as excellent/very good, good, and not so good/poor. Self-reported hypertension was coded as yes if a participant had ever been told to have high blood pressure/hypertension or was taking any prescribed medicine for hypertension. Self-reported hypercholesterolemia was coded as yes if a person had been told by a doctor to have a high cholesterol level or was taking any prescribed medicine to lower blood cholesterol. Self-reported diabetes was coded as yes if an individual was told by a doctor to have diabetes or was taking insulin or diabetic pills to lower blood sugar.

\section{Statistical analyses}

Weighted means and proportions were used for descriptive statistics. Linear regression was used to model the association between physical activity and continuous vitamin D levels. Objective physical activity was expressed as mean counts/minute, minutes/day spent in moderate-to-vigorous activities, and physical activity categories ( $\geq 150 \mathrm{~min} /$ week of moderate-to-vigorous activities versus less). Self-reported leisure-time physical activity was expressed as minutes per day in different intensities (moderate and vigorous) as well as indoor/outdoor categories. Logistic regression analysis was used to model the odds ratio (OR) of having a vitamin D deficiency based on the physical activity category. All results are adjusted for sex, age, ethnicity, PIR, education, marital status, season/region, alcohol intake, smoking, BMI, general health status, daily milk intake, vitamin D supplement intake, hypertension, hypercholesterolemia, and diabetes. For self-reported physical activity as exposure, the fully adjusted models also include adjustment for other physical activities (e.g., for outdoor activities when indoor activities are used in the analyses).

For objective physical activity, adjusted means were calculated by quartiles of physical activity levels (counts/ 
minute and mean minutes spent in moderate and vigorous activities) and by physical activity categories (insufficiently active and sufficiently active). Linear hypothesis testing was used to look at the trends of the adjusted means by physical activity level.

The regression analyses were also stratified individually by sex, age category (18-39, 40-64, and $\geq 65$ years), ethnicity, BMI category, vitamin D supplement intake (yes/ no), and season/region (data assessed in northern states during summer and in southern states during winter) while adjusting for the other variables. Interactions between the physical activity variables and these variables were tested in order to investigate whether associations between physical activity and vitamin D levels are different in the respective stratification groups. All analyses were weighted according to the NHANES guidelines [29] in order to account for the complex survey design, survey non-response, and post-stratification. A two-sided $p<0.05$ was considered as statistically significant. The analyses were performed using STATA version 12 (Stata Corporation, College Station, TX, USA).

\section{Results}

The mean age was 47.7 years, and the proportion of women was $51.6 \%$. Table 1 shows the characteristics of the study participants by physical activity category. Women were more likely to be insufficiently active. Mean age, BMI, and waist circumference were higher in insufficiently active individuals compared with sufficiently active ones. More sufficiently active individuals had a postsecondary education. Vitamin D serum levels were higher in sufficiently active individuals. Obviously, all physical activity variables were higher in sufficiently active individuals. Sufficiently active individuals were more likely to drink more than one alcoholic drink per week, had a better health status, and were less likely to have hypertension, hypercholesterolemia, and diabetes.

Table 2 (left side) displays the results of the linear regression analyses for objectively measured mean minutes spent in moderate-to-vigorous activities per day stratified by sex, age group, ethnicity, weight status, vitamin D supplement intake, and season/region. According to the fully adjusted model, an increase of $10 \mathrm{~min}$ of moderate-tovigorous activities per day was associated with a significant increase in circulating vitamin D of $0.32 \mathrm{ng} / \mathrm{ml}(95 \% \mathrm{CI}$ $0.17,0.48$ ), and an increase of 100 counts/minute with a statistically significant increase in vitamin D concentration of $0.75 \mathrm{ng} / \mathrm{ml}$ (95\% CI 0.53, 0.96) (Electronic Supplementary Material Table 1). Compared with being insufficiently active, individuals categorized as sufficiently active had on average $1.29 \mathrm{ng} / \mathrm{ml}$ (95\% CI 0.62, 1.96) higher vitamin D levels (Electronic Supplementary Material 1). According to the stratified analyses and the statistically significant interaction terms for these analyses, the associations were stronger in women than in men. There also seemed to be some effect modification for different ethnicities. However, there were no indications for effect modification by age group, weight status, and season/region. Stratified by intake of vitamin D supplements (yes/ no), there were significant associations between physical activity and vitamin D serum levels only for individuals not taking any vitamin D supplement with indications for effect modification depending on the physical activity variables (Table 2, Electronic Supplementary Material Table 1).

Using physical activity data of those individuals having at least 1 day of accelerometer data with at least 8 valid hours for sensitivity analyses $(n=8,178)$ did not considerably change the results (data not shown). The associations were generally slightly weaker (exposure less precise) and confidence intervals slightly smaller (larger sample size).

Table 2 (right side) presents the OR of being vitamin D deficient when being insufficiently active. Being insufficiently active was associated with a significant OR of 1.32 (95\% CI 1.11, 1.57) for having vitamin D deficiency. Again, the associations were statistically significant for individuals not taking any vitamin D supplements but not for those taking these supplements with indications for effect modification.

Mean vitamin D serum levels in individuals with different physical activity levels according to accelerometer data are displayed in Fig. 1. Adjusted means were higher in the third and fourth quartile of mean counts/minute and minutes spent in moderate-to-vigorous physical activity compared with the first quartile. Linear hypothesis testing revealed highly significant trends for vitamin $\mathrm{D}$ levels by physical activity level $(p<0.001$ for all three physical activity variables).

Table 3 presents the associations between self-reported indoor, outdoor, and total leisure-time physical activity and vitamin D serum concentration. A table for self-reported moderate- and vigorous-intensity leisure-time physical activities is shown in Electronic Supplementary Material Table 2. The associations for self-reported total leisuretime physical activity (Table 3) were smaller than those for objectively measured physical activity (Table 2). Furthermore, there were some differences when looking at the stratified analyses. Indications for effect modification were present for ethnicity, but not for sex, age, and vitamin D supplement intake. Unexpectedly, associations were not stronger for outdoor compared with indoor leisure-time activities in the fully adjusted models for the total sample and for most subgroups (Table 3 ). 
Table 1 Characteristics of participants, by objectively measured physical activity category (weighted according to the NHANES guidelines), NHANES 2003-2006

\begin{tabular}{|c|c|c|}
\hline & Insufficiently active $^{\mathrm{a}}$ & Sufficiently active $^{\mathrm{b}}$ \\
\hline$n$ (unweighted) & 3,924 & 2,446 \\
\hline Proportion $(\%)$ & 61.6 & 38.4 \\
\hline \multicolumn{3}{|l|}{ Gender $(\%)$} \\
\hline Men & 37.9 & 62.9 \\
\hline Women & 62.1 & 37.1 \\
\hline Age (years), mean (SE) & $52.6(0.57)$ & $41.0(0.44)$ \\
\hline \multicolumn{3}{|l|}{ Ethnicity (\%) } \\
\hline Non-Hispanic white & 74.7 & 73.2 \\
\hline Non-Hispanic black & 10.2 & 9.0 \\
\hline Mexican American & 6.5 & 9.6 \\
\hline Other & 8.6 & 8.2 \\
\hline \multicolumn{3}{|l|}{ Marital status (\%) } \\
\hline Married/living together & 66.2 & 67.9 \\
\hline Single (widowed, separated, divorced) & 33.7 & 32.1 \\
\hline Missing information & 0.1 & 0.0 \\
\hline \multicolumn{3}{|l|}{ Poverty index ratio $(\%)$} \\
\hline Below poverty & 9.9 & 8.6 \\
\hline At or above poverty & 85.0 & 88.8 \\
\hline Missing information & 5.1 & 2.6 \\
\hline \multicolumn{3}{|l|}{ Education $(\%)$} \\
\hline High school or lower & 44.6 & 34.4 \\
\hline Post-secondary (college or higher) & 54.1 & 62.0 \\
\hline Missing information & 1.3 & 3.6 \\
\hline Body mass index $\left(\mathrm{kg} / \mathrm{m}^{2}\right)$, mean (SE) & $29.3(0.18)$ & $26.9(0.18)$ \\
\hline Waist circumference $(\mathrm{cm})$, mean (SE) & $99.9(0.42)$ & $93.8(0.42)$ \\
\hline \multicolumn{3}{|l|}{ BMI category $(\%)$} \\
\hline Normal weight & 28.2 & 39.3 \\
\hline Overweight & 32.5 & 37.4 \\
\hline Obese & 38.1 & 23.1 \\
\hline Missing information & 1.2 & 0.2 \\
\hline Vitamin D serum level (ng/ml), mean (SE) & $22.8(0.35)$ & $25.2(0.50)$ \\
\hline \multicolumn{3}{|l|}{ Vitamin D supplement intake } \\
\hline Mean intake (mcg/day), mean (SE) & $1.2(0.07)$ & $0.7(0.06)$ \\
\hline Taking any $(\%)$ & 14.7 & 11.0 \\
\hline \multicolumn{3}{|l|}{ Objective physical activity, mean (SE) } \\
\hline Mean total (counts/min) & $232(2.03)$ & $440(2.93)$ \\
\hline Moderate and vigorous (min/day) & $9.0(0.15)$ & $43.4(0.49)$ \\
\hline \multicolumn{3}{|l|}{ Self-reported physical activity, mean (SE) } \\
\hline Total leisure time (min/day) & $19.0(0.79)$ & $38.6(1.22)$ \\
\hline Moderate leisure time (min/day) & $14.2(0.68)$ & $23.8(0.89)$ \\
\hline Vigorous leisure time (min/day) & $4.9(0.32)$ & $14.8(0.68)$ \\
\hline Leisure-time indoor (min/day) & $6.8(0.33)$ & $13.6(0.82)$ \\
\hline Leisure-time outdoor (min/day) & $12.3(0.73)$ & $24.9(0.89)$ \\
\hline \multicolumn{3}{|l|}{ Smoking status (\%) } \\
\hline Current non-smoker & 78.8 & 79.1 \\
\hline Current smoker & 21.2 & 20.9 \\
\hline \multicolumn{3}{|l|}{ Alcohol intake (\%) } \\
\hline No drinks & 40.5 & 28.6 \\
\hline$\leq 1 \mathrm{drink} /$ week & 33.9 & 28.4 \\
\hline
\end{tabular}


Table 1 continued

\begin{tabular}{lll}
\hline & Insufficiently active & Sufficiently active \\
\hline$>1$ drink/week & 25.6 & 43.0 \\
Milk consumption past 30 days (\%) & & \\
Less than daily & 55.6 & 55.3 \\
Daily & 44.4 & 44.7 \\
Health status (\%) & & \\
Excellent or very good & 39.4 & 55.3 \\
Good & 37.0 & 31.5 \\
Not so good or bad & 18.7 & 8.5 \\
Missing information & 4.9 & 4.7 \\
Hypertension (\%) & & \\
No hypertension & 60.5 & 81.4 \\
Hypertension & 39.0 & 18.2 \\
Missing information & 0.5 & 0.4 \\
Hypercholesterolemia (\%) & & 40.4 \\
No hypercholesterolemia & 42.3 & 24.3 \\
Hypercholesterolemia & 34.9 & 35.3 \\
Never checked or missing & 22.7 & 97.1 \\
Diabetes (\%) & & 2.9 \\
No diabetes & 88.7 & \\
Diabetes & 11.3 & \\
\hline
\end{tabular}

a Achieving $<150$ min per week of moderate-to-vigorous activities

b Achieving $\geq 150$ min per week of moderate-to-vigorous activities

\section{Discussion}

In the present analyses based on USA nationally representative data, findings indicate that physical activity is associated with higher levels of serum vitamin D. The associations were stronger for objectively measured than those for self-reported physical activity. Interestingly, the associations were not stronger for self-reported outdoor compared with indoor physical activity. Statistically significant associations were observed in some subgroups, while nonsignificant tendencies were observed in the other subgroups. According to objective physical activity, the associations disappeared for individuals taking vitamin D supplements. There are some indications that the associations were stronger in women according to objective physical activity data.

An important determinant of the associations between physical activity and circulating vitamin D concentration is sun exposure during outdoor physical activity. Kluczynski et al. came to the conclusion that these associations largely reflect the effect of sunlight exposure during outdoor physical activity because they found significant associations only for summer/fall, but not for winter/spring [24]. However, the fact that in the present study the associations were not stronger for self-reported outdoor compared with indoor physical activity indicates that other effects independent of sun exposure may play a role. This is supported by a French study reporting an association between physical activity and vitamin D even after adjustment for sun exposure and practice of outdoor hobbies or sports [41]. The study found $15 \%$ higher vitamin $\mathrm{D}$ concentrations in individuals practicing at least $1 \mathrm{~h}$ per day walking equivalent compared with irregular physical activity. Furthermore, the correlation between indoor and outdoor physical activities of 0.29 indicates that active people are usually active both inside and outside. A review reported elevated levels of vitamin D metabolites in athletes [42] and hypothesized that factors other than sun exposure that are known to be altered by physical activity could contribute to the observed associations. Firstly, a decrease in serum phosphate may influence vitamin D serum levels (phosphate suppresses the production of activated vitamin $\mathrm{D}$ via a negative feedback system [43]) [42]. Secondly, a decrease in ionized calcium during exercise may stimulate parathyroid hormone secretion and thereby activate renal calcitriol synthesis (active form of vitamin D) [42]. In the present study, misclassification of indoor and outdoor activities may also play a role, but it is unlikely that misclassification alone accounted for the unexpected results regarding indoor and outdoor activities. Furthermore, indoor physical activity may be more structured 
Table 2 Associations between objectively measured physical activity and vitamin D serum, by sex, age category, ethnicity, weight status, vitamin D supplement intake, and season/region; NHANES 2003-2006

\begin{tabular}{|c|c|c|c|c|c|c|}
\hline & \multicolumn{3}{|c|}{$\begin{array}{l}\text { Mean minutes per day spent in moderate-to- } \\
\text { vigorous activities versus vitamin D serum } \\
\text { (continuous variables) }\end{array}$} & \multicolumn{3}{|c|}{$\begin{array}{l}\text { Odds ratio }(\mathrm{OR}) \text { of being vitamin } \mathrm{D} \text { deficient } \\
(<20 \mathrm{ng} / \mathrm{ml}) \text { if being insufficiently active }(<150 \mathrm{~min} \\
\text { of moderate-to-vigorous physical activity per week) }\end{array}$} \\
\hline & $\beta^{\mathrm{a}}$ & $95 \% \mathrm{CI}$ & $p$ interaction & OR & $95 \% \mathrm{CI}$ & $p$ interaction \\
\hline Total sample & 0.032 & $0.017,0.048$ & & 1.32 & $1.11,1.57$ & \\
\hline Men & 0.031 & $0.016,0.046$ & & 1.30 & $1.06,1.59$ & \\
\hline Women & 0.047 & $0.020,0.074$ & 0.003 & 1.37 & $1.02,1.85$ & 0.125 \\
\hline $18-39$ years & 0.051 & $0.032,0.070$ & & 1.55 & $1.11,2.18$ & \\
\hline $40-64$ years & 0.020 & $-0.001,0.041$ & 0.450 & 1.27 & $1.01,1.60$ & 0.744 \\
\hline$\geq 65$ years & 0.058 & $0.032,0.084$ & 0.067 & 1.04 & $0.73,1.47$ & 0.318 \\
\hline Non-Hispanic white & 0.031 & $0.010,0.052$ & & 1.22 & $0.99,1.50$ & \\
\hline Non-Hispanic black & 0.031 & $0.010,0.052$ & 0.029 & 1.70 & $1.13,2.55$ & 0.103 \\
\hline Mexican American & 0.022 & $0.002,0.041$ & 0.070 & 1.46 & $1.07,1.98$ & 0.946 \\
\hline Other & 0.022 & $-0.013,0.057$ & 0.361 & 1.06 & $0.65,1.73$ & 0.647 \\
\hline Normal weight & 0.043 & $0.020,0.066$ & & 1.27 & $0.90,1.78$ & \\
\hline Overweight & 0.025 & $0.007,0.044$ & 0.917 & 1.11 & $0.83,1.48$ & 0.943 \\
\hline Obese & 0.033 & $0.011,0.054$ & 0.296 & 1.68 & $1.26,2.23$ & 0.067 \\
\hline Vitamin D supplement intake & 0.016 & $-0.028,0.060$ & & 0.72 & $0.34,1.53$ & \\
\hline No vitamin D supplement intake & 0.034 & $0.017,0.052$ & 0.086 & 1.38 & $1.18,1.62$ & 0.020 \\
\hline Winter (= southern states) & 0.046 & $0.023-0.068$ & & 1.46 & $1.12,1.90$ & \\
\hline Summer (= northern states) & 0.021 & $0.002-0.040$ & 0.399 & 1.21 & $0.94,1.54$ & 0.452 \\
\hline
\end{tabular}

All analyses adjusted for sex, age, ethnicity, poverty income ratio, education, marital status, season, alcohol intake, smoking, weight status, general health status, daily milk intake, vitamin D supplement intake, hypertension, hypercholesterolemia, and diabetes

${ }^{a} \beta$ from linear regression analyses is interpreted as the increase in circulating vitamin $\mathrm{D}$ concentration (ng/ml) per additional minute/day of physical activity

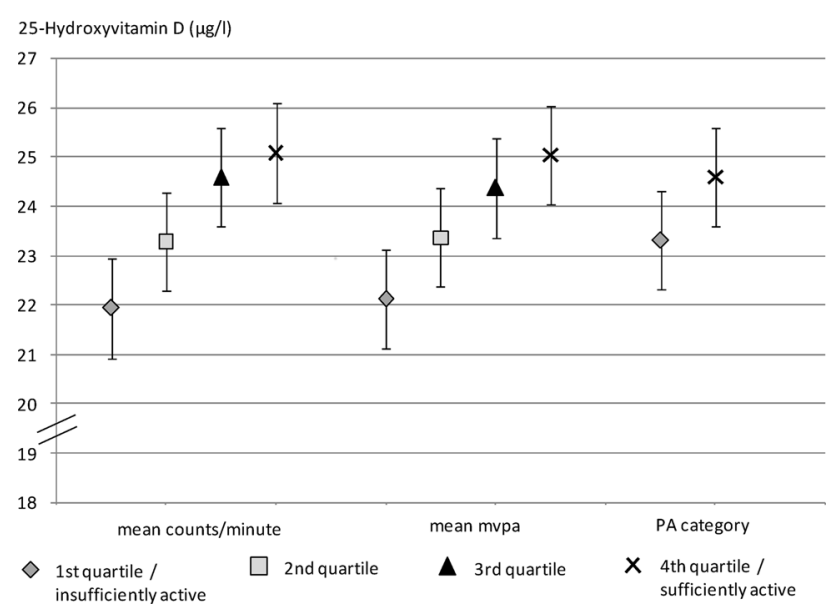

Fig. 1 Adjusted means of 25-hydroxyvitamin D by objectively measured physical activity levels. $P A$ physical activity, mvpa moderate-to-vigorous physical activity. Mean mvpa in minutes per day. PA categories: insufficiently active, $<150 \mathrm{~min}$ per week of moderate-to-vigorous activities; sufficiently active, $\geq 150 \mathrm{~min}$ per week of moderate-to-vigorous activities

and therefore easier to recall, leading to smaller measurement errors for indoor than those for outdoor activities.
The stronger associations observed for objectively measured compared with self-reported physical activity may be due to measurement error in the self-report instrument which attenuates the true association. Furthermore, accelerometers are especially useful for measuring ambulatory activity such as walking and jogging [44], activities that may be performed outside to a large extent. Therefore, physical activity measured by accelerometers may to a larger part reflect outdoor activity than self-reported physical activity.

Similar to our results, Scragg and Camargo reported associations between self-reported physical activity and vitamin D [23]. These analyses based on NHANES III data showed stronger associations for outdoor than those for indoor activities, which was not replicated in our analyses using more recent NHANES data. However, the slightly stronger associations observed for vigorous-intensity compared with moderate-intensity activities (Electronic Supplementary Material Table 2) were comparable. Furthermore, both studies reported associations between physical activity and vitamin D also in older individuals, even though the capacity to synthesize vitamin D from sun exposure decreases with age [45] (but is still present and 


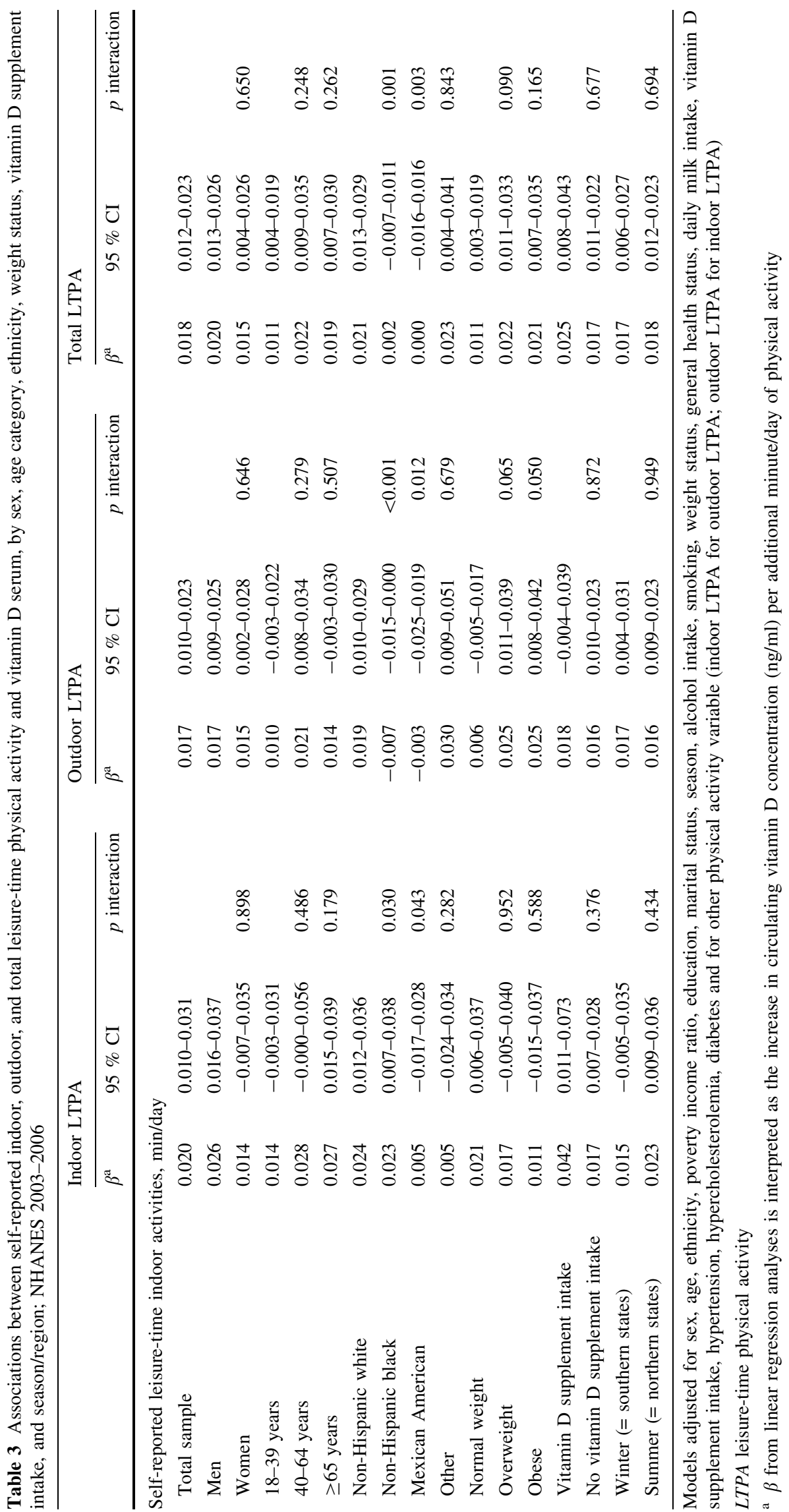


effective). This supports the indication that effects of physical activity other than sun exposure may also play a role. For objective physical activity, we also found associations with vitamin $\mathrm{D}$ for the different ethnicities, even though the capacity to synthesize vitamin D depends on skin color [46]. For self-reported physical activity, the associations were not significant for non-Hispanic blacks and Mexican Americans with the exception of indoor activity for non-Hispanic blacks and for vigorous physical activity in Mexican Americans in our study. Although Scragg and Camargo also reported associations between physical activity and circulating vitamin $\mathrm{D}$ concentration for different ethnicities, the levels of vitamin D were much lower in Mexican Americans and non-Hispanic blacks compared with non-Hispanic whites [23].

Our study is also in line with other studies reporting correlations between physical activity and vitamin D [24, 25]. While these studies did not distinguish between different intensities of physical activity and indoor/outdoor activity, Kluczynski et al. included self-reported sunlight exposure as a confounder in the analyses [24]. Although the inclusion of this confounder weakened the association between physical activity and vitamin D, it was still significant. An Australian study reported that physical activity contributed $4 \%$ to the explained variance in 25-hydroxyvitamin D (compared with $17 \%$ for season, $7 \%$ for vitamin D supplement intake, and $27 \%$ for the proportion of skin covered by clothing) [47].

For objective physical activity, associations stratified by vitamin D supplement intake were only statistically significant for individuals not taking any vitamin D supplements. In those taking supplements, the additional effect of physical activity on circulating vitamin $\mathrm{D}$ concentrations was probably not strong enough to reach statistical significance. Interestingly, the impact of supplementation was not observed in the analyses using self-reported physical activity data.

The strengths of our study are the large sample size representative of the US population and the use of both objective and self-reported physical activity data that could be categorized as indoor and outdoor activities. Furthermore, a variety of socio-demographic, behavioral, and health-related variables were included as potential confounders. The study has also some limitations. The design is cross-sectional, and thus, no inference about cause and effect is possible. However, it is rather unlikely that individuals participate in less physical activity because they have low vitamin D serum levels. We had no measure of sun exposure and no detailed information on where people lived. As sun exposure is an important determinant of vitamin D serum levels, the lack of a precise measure of sun exposure is a major limitation of this and other studies. Furthermore, measurement error particularly regarding the self-reported physical activity data and the classification of indoor and outdoor is likely to be present. Several activities can be performed both indoor and outdoor, and it is likely that this also depends on where people live (e.g., outdoor swimming in the south and in coastal regions, indoor swimming in the north). Accelerometers had to be taken off during water-based activities such as swimming which can result in an underestimation of true activity.

In conclusion, physical activity has several positive effects on human health. This paper adds evidence that one of the positive effects is a higher level of serum vitamin D. This association was observed in several subgroups according to age, sex, ethnicity, and BMI status. The findings in the present analyses that associations for outdoor leisuretime physical activities were not stronger than those for indoor activities need further investigation.

Conflict of interest The authors declare that they have no conflict of interest.

\section{References}

1. Zittermann A, Iodice S, Pilz S, Grant WB, Bagnardi V, Gandini S (2012) Vitamin D deficiency and mortality risk in the general population: a meta-analysis of prospective cohort studies. Am J Clin Nutr 95(1):91-100. doi:10.3945/ajcn.111.014779

2. Rohrmann S, Braun J, Bopp M, Faeh D (2013) Inverse association between circulating vitamin D and mortality-Dependent on sex and cause of death? Nutr Metab Cardiovasc Dis. doi:10. 1016/j.numecd.2013.05.005

3. Deng X, Song Y, Manson JE, Signorello LB, Zhang SM, Shrubsole MJ et al (2013) Magnesium, vitamin D status and mortality: results from US National Health and Nutrition Examination Survey (NHANES) 2001 to 2006 and NHANES III. BMC Med 11:187. doi:10.1186/1741-7015-11-187

4. Sempos CT, Durazo-Arvizu RA, Dawson-Hughes B, Yetley EA, Looker AC, Schleicher RL et al (2013) Is there a reverse J-shaped association between 25-hydroxyvitamin D and all-cause mortality? Results from the U.S. nationally representative NHANES. J Clin Endocrinol Metab 98(7):3001-3009. doi:10.1210/jc.20131333

5. Liu L, Chen M, Hankins SR, Nunez AE, Watson RA, Weinstock PJ et al (2012) Serum 25-hydroxyvitamin D concentration and mortality from heart failure and cardiovascular disease, and premature mortality from all-cause in United States adults. Am J Cardiol 110(6):834-839. doi:10.1016/j.amjcard.2012.05.013

6. Chowdhury R, Kunutsor S, Vitezova A, Oliver-Williams C, Chowdhury S, Kiefte-de-Jong JC et al (2014) Vitamin D and risk of cause specific death: systematic review and meta-analysis of observational cohort and randomised intervention studies. BMJ 348:g1903. doi:10.1136/bmj.g1903

7. Yin L, Ordonez-Mena JM, Chen T, Schottker B, Arndt V, Brenner H (2013) Circulating 25-hydroxyvitamin D serum concentration and total cancer incidence and mortality: a systematic review and meta-analysis. Prev Med 57(6):753-764. doi:10.1016/ j.ypmed.2013.08.026

8. Jenab M, Bueno-de-Mesquita HB, Ferrari P, van Duijnhoven FJ, Norat T, Pischon T et al (2010) Association between pre-diagnostic circulating vitamin $\mathrm{D}$ concentration and risk of colorectal 
cancer in European populations: a nested case-control study. BMJ 340:b5500. doi:10.1136/bmj.b5500

9. Abbas S, Linseisen J, Slanger T, Kropp S, Mutschelknauss EJ, Flesch-Janys D et al (2008) Serum 25-hydroxyvitamin D and risk of post-menopausal breast cancer-results of a large case-control study. Carcinogenesis 29(1):93-99. doi:10.1093/carcin/bgm240

10. Kendrick J, Targher G, Smits G, Chonchol M (2009) 25-Hydroxyvitamin D deficiency is independently associated with cardiovascular disease in the Third National Health and Nutrition Examination Survey. Atherosclerosis 205(1):255-260. doi:10. 1016/j.atherosclerosis.2008.10.033

11. Kabadi SM, Lee BK, Liu L (2012) Joint effects of obesity and vitamin D insufficiency on insulin resistance and type 2 diabetes: results from the NHANES 2001-2006. Diabetes Care 35(10):2048-2054. doi:10. 2337/dc12-0235

12. Kabadi SM, Liu L, Auchincloss AH, Zakeri IF (2013) Multivariate path analysis of serum 25 -hydroxyvitamin d concentration, inflammation, and risk of type 2 diabetes mellitus. Dis Markers 35(3):187-193. doi:10.1155/2013/497256

13. Freedman DM, Looker AC, Abnet CC, Linet MS, Graubard BI (2010) Serum 25-hydroxyvitamin D and cancer mortality in the NHANES III study (1988-2006). Cancer Res 70(21):8587-8597. doi:10.1158/0008-5472.CAN-10-1420

14. Scragg RK, Camargo CA Jr, Simpson RU (2010) Relation of serum 25-hydroxyvitamin D to heart rate and cardiac work (from the National Health and Nutrition Examination Surveys). Am J Cardiol 105(1):122-128. doi:10.1016/j.amjcard.2009.08.661

15. Physical Activity Guidelines Advisory Committee (2008) Physical activity guidelines advisory committee report 2008. U.S. Department of Health and Human Services, Washington

16. World Health Organization (2009) Global health risks: mortality and burden of disease attributable to selected major risks. WHO, Geneva

17. Zhao G, Li C, Ford ES, Fulton JE, Carlson SA, Okoro CA et al (2013) Leisure-time aerobic physical activity, muscle-strengthening activity and mortality risks among US adults: the NHANES linked mortality study. Br J Sports Med. doi:10.1136/bjsports2013-092731

18. Richard A, Martin BW, Wanner M, Eichholzer M, Rohrmann S (2014) Effects of leisure-time and occupational physical activity on total mortality risk in NHANES III according to sex, ethnicity, central obesity and age. J Phys Act Health. doi:10.1123/jpah. 2013-0198

19. Metzger JS, Catellier DJ, Evenson KR, Treuth MS, Rosamond WD, Siega-Riz AM (2008) Patterns of objectively measured physical activity in the United States. Med Sci Sports Exerc 40(4):630-638. doi:10.1249/MSS.0b013e3181620ebc

20. Troiano RP, Berrigan D, Dodd KW, Masse LC, Tilert T, McDowell M (2008) Physical activity in the United States measured by accelerometer. Med Sci Sports Exerc 40(1):181-188

21. Matthews CE, Chen KY, Freedson PS, Buchowski MS, Beech BM, Pate RR et al (2008) Amount of time spent in sedentary behaviors in the United States, 2003-2004. Am J Epidemiol 167(7):875-881. doi:10.1093/aje/kwm390

22. Schuna JM Jr, Johnson WD, Tudor-Locke C (2013) Adult selfreported and objectively monitored physical activity and sedentary behavior: NHANES 2005-2006. Int J Behav Nutr Phys Act 10(1):126. doi:10.1186/1479-5868-10-126

23. Scragg R, Camargo CA Jr (2008) Frequency of leisure-time physical activity and serum 25-hydroxyvitamin D levels in the US population: results from the Third National Health and Nutrition Examination Survey. Am J Epidemiol 168(6):577-586. doi:10.1093/aje/kwn163 discussion 87-91

24. Kluczynski MA, Lamonte MJ, Mares JA, Wactawski-Wende J, Smith AW, Engelman CD et al (2011) Duration of physical activity and serum 25-hydroxyvitamin D status of postmenopausal women. Ann Epidemiol 21(6):440-449. doi:10.1016/j.annepi dem.2010.11.011

25. Brock K, Huang WY, Fraser DR, Ke L, Tseng M, StolzenbergSolomon R et al (2010) Low vitamin D status is associated with physical inactivity, obesity and low vitamin D intake in a large US sample of healthy middle-aged men and women. J Steroid Biochem Mol Biol 121(1-2):462-466. doi:10.1016/j.jsbmb.2010. 03.091

26. McCullough ML, Weinstein SJ, Freedman DM, Helzlsouer K, Flanders WD, Koenig K et al (2010) Correlates of circulating 25-hydroxyvitamin D: cohort Consortium Vitamin D Pooling Project of Rarer Cancers. Am J Epidemiol 172(1):21-35. doi:10. 1093/aje/kwq113

27. Millen AE, Wactawski-Wende J, Pettinger M, Melamed ML, Tylavsky FA, Liu S et al (2010) Predictors of serum 25-hydroxyvitamin D concentrations among postmenopausal women: the Women's Health Initiative Calcium plus Vitamin D clinical trial. Am J Clin Nutr 91(5):1324-1335. doi:10.3945/ajcn.2009.28908

28. Tseng M, Giri V, Bruner DW, Giovannucci E (2009) Prevalence and correlates of vitamin D status in African American men. BMC Public Health 9:191. doi:10.1186/1471-2458-9-191

29. Johnson CL, Paulose-Ram R, Ogden CL, Carroll MD, KruszonMoran D, Dohrmann SM et al (2013) National health and nutrition examination survey: analytic guidelines, 1999-2010. Vital Health Stat 2(161):1-7, 7-10

30. Hendelman D, Miller K, Baggett C, Debold E, Freedson P (2000) Validity of accelerometry for the assessment of moderate intensity physical activity in the field. Med Sci Sports Exerc 32(9 Suppl):S442-S449

31. Melanson EL Jr, Freedson PS (1995) Validity of the Computer Science and Applications, Inc. (CSA) activity monitor. Med Sci Sports Exerc 27(6):934-940

32. Brage S, Wedderkopp N, Franks PW, Andersen LB, Froberg K (2003) Reexamination of validity and reliability of the CSA monitor in walking and running. Med Sci Sports Exerc 35(8):1447-1454. doi:10.1249/01.MSS.0000079078.62035.EC

33. Matthews CE (2005) Calibration of accelerometer output for adults. Med Sci Sports Exerc 37(11 Suppl):S512-S522

34. Pfeiffer C Laboratory procedure manual. 25-Hydroxyvitamin D in Serum. NHANES 2003-2004

35. Pfeiffer C (2008) Laboratory procedure manual. 25-Hydroxyvitamin D in Serum. NHANES 2005-2006

36. Forrest KY, Stuhldreher WL (2011) Prevalence and correlates of vitamin D deficiency in US adults. Nutr Res 31(1):48-54. doi:10. 1016/j.nutres.2010.12.001

37. Wallace TC, Reider C, Fulgoni VL (2013) Calcium and Vitamin D disparities are related to gender, age, race, household income level, and weight classification but not vegetarian status in the United States: analysis of the NHANES 2001-2008 data set. J Am Coll Nutr 32(5):321-330. doi:10.1080/07315724.2013.839905

38. O'Neil CE, Keast DR, Fulgoni VL, Nicklas TA (2012) Food sources of energy and nutrients among adults in the US: NHANES 2003-2006. Nutrients 4(12):2097-2120. doi:10.3390/nu4122097

39. Samuel L, Borrell LN (2013) The effect of body mass index on optimal vitamin D status in U.S. adults: the National Health and Nutrition Examination Survey 2001-2006. Ann Epidemiol 23(7):409-414. doi:10.1016/j.annepidem.2013.05.011

40. Sabanayagam C, Shankar A, Somasundaram S (2012) Serum vitamin D level and prehypertension among subjects free of hypertension. Kidney Blood Press Res 35(2):106-113. doi:10.1159/ 000330716

41. Touvier M, Deschasaux M, Montourcy M, Sutton A, Charnaux N, Kesse-Guyot E et al (2015) Determinants of vitamin D status in Caucasian adults: influence of sun exposure, dietary intake, sociodemographic, lifestyle, anthropometric, and genetic factors. J Invest Dermatol 135(2):378-388. doi:10.1038/jid.2014.400 
42. Maimoun L, Sultan C (2009) Effect of physical activity on calcium homeostasis and calciotropic hormones: a review. Calcif Tissue Int 85(4):277-286. doi:10.1007/s00223-009-9277-z

43. Fukumoto S (2014) Phosphate metabolism and vitamin D. Bonekey Rep 3:497. doi:10.1038/bonekey.2013.231

44. Corder K, Brage S, Ekelund U (2007) Accelerometers and pedometers: methodology and clinical application. Curr Opin Clin Nutr Metab Care 10(5):597-603. doi:10.1097/MCO.0b013e328285d883

45. Lips P (2001) Vitamin D deficiency and secondary hyperparathyroidism in the elderly: consequences for bone loss and fractures and therapeutic implications. Endocr Rev 22(4):477-501. doi:10.1210/ edrv.22.4.0437

46. Clemens TL, Adams JS, Henderson SL, Holick MF (1982) Increased skin pigment reduces the capacity of skin to synthesise vitamin D3. Lancet 1(8263):74-76

47. Kimlin MG, Lucas RM, Harrison SL, van der Mei I, Armstrong BK, Whiteman DC et al (2014) The contributions of solar ultraviolet radiation exposure and other determinants to serum 25-Hydroxyvitamin D concentrations in Australian adults: the AusD Study. Am J Epidemiol. doi:10.1093/aje/kwt446 